Short Report

\title{
Biochemical derangements prior to emergency laparotomy at Queen Elizabeth Central Hospital, Blantyre, Malawi: A cross-sectional pilot study
}

\author{
Mark G. Williams \\ Department of Surgery, North Devon District Hospital, Barnstaple, United Kingdom
}

Correspondence: m.williams13@nhs.net

Background

Abstract

The aim of this study was to examine biochemical investigations not routinely performed prior to emergency laparotomy in patients at Queen Elizabeth Central Hospital, a low-resource public hospital in Blantyre, Malawi..

Methods

A prospective cross-sectional study of adults $(\mathrm{N}=15)$ needing emergency laparotomy over a 4-week period were studied at Queen Elizabeth Central Hospital. Biochemical investigations, not routinely performed for economic reasons, were performed preoperatively; these included sodium, potassium, chloride, carbon dioxide, urea, and calcium levels.

Results

Gastrointestinal pathology was predominant among the emergency laparotomies performed. Large bowel obstruction and bowel perforation secondary to typhoid were most frequent. Clinically significant biochemical derangements among the study patients were as follows: cases of moderate-tosevere hypokalaemia $(n=2)$, severe hyponatraemia $(n=1)$, moderate hypernatraemia $(n=1)$, and severe hypocalcaemia $(n=1)$. The most frequent abnormalities seen were uraemia and hypochloraemia $(n=11)$.

Conclusions

Accurate electrolyte estimation in critically ill preoperative patients is desireable for optimal perioperative management but frequently absent in resourcechallenged environments.

\section{Introduction}

An emergency laparotomy (EL) is considered a high-risk surgical procedure, frequently contributing to significant morbidity and mortality. A clear imperative exists to mitigate these risks. In high-income countries, emphasis is placed on streamlining preoperative diagnosis (aided by comprehensive laboratory and radiological investigations) and timely transfer to theatre. ${ }^{1}$

Patients frequently present to Queen Elizabeth Central Hospital (QECH) in Blantyre, Malawi, after significant delays, with disease progression and life-threatening complications. Samuel et al. reported a $15 \%$ postoperative hospital mortality rate among patients with peritonitis presenting to Kamuzu Central Hospital in Lilongwe. ${ }^{2}$ At QECH, EL patients routinely have preoperative full blood count and blood grouping tests done, but lack a biochemical profile. This paper seeks to highlight the importance of ascertaning EL patients' biochemical profile to aid optimal perioperative management.

\section{Methods}

This was a prospective cross-sectional study of adult patients presenting to QECH, with an acute abdomen, over a 4-week period (April to May 2015). Venepuncture was performed preoperatively for urgent cases, defined as those requiring EL within 24 hours of admission. The Malawi-LiverpoolWellcome Trust laboratories procesed samples, with results available the next working day. Nonoperative acute abdomens and paediatric cases were excluded.

\section{Results}

Data were collected for 9 males and 6 females $(\mathrm{N}=15)$, with a median age of 37 years (range 19 to 89 years). Table 1 demonstrates the frequency of biochemical derangement in this series. Cases of severe hyponatraemia $(119 \mathrm{mmol} / \mathrm{L})$, moderate hypernatraemia $(153 \mathrm{mmol} / \mathrm{L})$, and moderate and severe hypokalaemia $(3.0$ and $2.6 \mathrm{mmol} / \mathrm{L})$ were observed. All 5 cases of hyperkalaemia were mild $(<6.0 \mathrm{mmol} / \mathrm{L})$. The most frequent abnormalities detected were uraemia and hypochloraemia. Severe uraemia was noted in 1 patient $(56.8$ $\mathrm{mmol} / \mathrm{L})$. Of the 3 hypercalcemia cases, all were mild; 1 of the 5 hypocalcaemia cases was severe $(1.56 \mathrm{mmol} / \mathrm{L})$. Table 2 shows that large bowel obstruction and bowel perforation secondary to typhoid were the most frequent pathologies found.

Table 1: Frequency of abnormal biochemistry results among laparotomy patients $(N=15)$

\begin{tabular}{lccc}
\hline $\begin{array}{l}\text { Biochemical test (reference } \\
\text { interval) }\end{array}$ & $\begin{array}{l}\text { Frequency of } \\
\text { abnormal results }\end{array}$ & $\begin{array}{l}\text { Frequency of results } \\
\text { below reference interval }\end{array}$ & $\begin{array}{l}\text { Frequency of results } \\
\text { above reference interval }\end{array}$ \\
\hline Sodium $(126-145 \mathrm{mmol} / \mathrm{L})$ & 3 & 2 & 1 \\
Potassium $(3.5-4.3 \mathrm{mmol} / \mathrm{L})$ & 7 & 2 & 5 \\
Calcium $(2.00-2.40 \mathrm{mmol} / \mathrm{L})$ & 8 & 5 & 3 \\
Carbon dioxide $(21-28 \mathrm{mmol} / \mathrm{L})$ & 8 & 6 & 2 \\
Urea $(2.1-7.1 \mathrm{mmol} / \mathrm{L})$ & 11 & 0 & 11 \\
Chloride $(105-116 \mathrm{mmol} / \mathrm{L})$ & 11 & 11 & 0
\end{tabular}


Table 2: Diagnoses made during laparotomy

\begin{tabular}{lc}
\hline Diagnosis & Frequency $(\mathbf{N}=\mathbf{1 5})$ \\
\hline Bowel obstruction & 4 \\
Typhoid perforation & 4 \\
Small bowel obstruction & 2 \\
Pseudo-obstruction & 1 \\
Compound ileocaecal volvulus & 1 \\
Appendicitis & 1 \\
Anastomotic leak & 1 \\
Died on theatre table & 1 \\
\hline
\end{tabular}

\section{Discussion}

All the patients in this series had gastrointestinal pathologies-diagnoses frequently associated with biochemical derangement. A substantial proportion displayed clinically significant derangements in their blood tests. These derangements can result in anorexia, nausea, muscle weakness, paralytic ileus, and cardiac conduction abnormalities, all or any of which can contribute to increased postoperative morbidity and mortality. ${ }^{3}$ Once identified, derangements, such as hypokalaemia and hypocalcaemia are readily corrected with supplementation. The cases of hyperkalaemia observed were mild, but without routine testing, severe hyperkalaemia $(>7 \mathrm{mmol} / \mathrm{L})$ cases, leading to life-threatening cardiac arrhythmias, may be missed. On the other hand, interpretation of mild hyperkalaemia cases requires acknowledgment of the tourniquet duration during venepuncture. That is, EL patients often have challenging venepuncture with prolonged tourniquet use resulting in haemoconcentration and subsequent erroneously increased potassium results, which can further obfuscate clinical decision-making.

Identification of an acute symptomatic hyponatraemia, a medical emergency, necessitates gradual correction to avoid adverse patient outcomes. Effective management of electrolyte abnormalities necessitates serial monitoring. Equally important in cases of hypernatraemia is correction over 48 to 72 hours, typically with 5\% dextrose. ${ }^{3}$

One of this study's limitations was that serum creatinine and lactate levels were not measured. Glasmacher et al. reported that point-of-care creatinine and lactate testing was feasible, practical, and well received by QECH staff, and that it was considered clinically useful in the care of sick obstetric patients. ${ }^{4}$ Patients who develop progressive renal failure retain solutes that are usually excreted by the kidneys. It is likely that many of the EL patients in this study had a degree of prerenal acute kidney injury, with the elevated urea results reflecting this process. Patients with deranged renal function, such as that seen in the study, require preoperative assessment for renal replacement therapy.

Samuel et al. demonstrated that generalised (versus localised) peritonitis, hypotension, tachycardia, and anaemia were significant risk factors for mortality in peritonitis patients. ${ }^{2}$ This pilot study highlights some of the severe electrolyte abnormalities in EL patients which, in a resource-poor environment, may never be investigated.

Another limitation of this study is the lack of patient-related outcome measures and mortality data. This limitation does not undermine the validity of the concerns engendered by the absence of biochemical investigations in EL patients in this setting.

Preoperative biochemical testing should be mandatory and would contribute to safer surgery, as failure to identify electrolyte derangements potentially contributes to medical emergencies to the further detriment of our patients. Serial monitoring is desirable to guide correction and avoidance of severe adverse outcomes.

The present study was not designed to assess the costeffectiveness, effects on patient outcome, or feasibility of routine testing. Implementation of routine biochemistry testing in a low-income country, such a Malawi, requires careful assessment at multiple sites. Henry et al. reported that greater than $75 \%$ of district hospitals surveyed in Malawi had inconsistent water and electricity supply. Other areas warranting attention include critical workforce levels, basic infrastructure, and service delivery. ${ }^{5}$ Prioritisation and allocation of resources, as well as lack of infrastructure may make implementation of routine biochemistry testing challenging in some settings, but their importance cannot be underestimated.

\section{Conclusions}

Accurate electrolyte estimation in the critically ill preoperative laparotomy patient is desirable for optimal perioperative management but frequently absent in resource-challenged environments.

\section{Competing interests}

The authors declare that they have no conflicts of interest.

\section{References}

1. The Royal College of Surgeons of England and Department of Health. Emergency abdominal surgery. 2013.

2. Samuel JC, Qureshi JS, Mulima G, Shores CG, Cairns BA, Charles AG. An Observational Study of the Etiology, clinical presentation and outcomes associated with peritonitis in Lilongwe, Malawi. World Journal of Emergency Surgery. 2011 Nov 8;6(1): 37. doi: 10.1186/1749-7922-637.

3. Rassam S, Counsell D. Perioperative electrolyte and fluid balance. Contin Educ Anaesth Crit Care Pain. 2005 Oct 5 (5): 161-165. doi: 10.1093/ bjaceaccp/mki043

4. Glasmacher S, Bonongwe P, Stones W. Point-of-care lactate and creatinine analysis for sick obstetric patients at Queen Elizabeth Central Hospital in Blantyre, Malawi: A feasibility study. Malawi Medical Journal. 2016 Mar; 28(1): 15-18

5. Henry JA, Frenkel E, Borgstein E, Mkandawire N, Goddia C. Surgical and anaesthetic capacity of hospitals in Malawi: key insights online. Health Policy Plan. 2015 Oct; 30(8): 985-994.. 